\title{
Application of Queuing Model in a Restaurant (A Case Study of Kalinga Restaurant)
}

\author{
Umar Muhammad Abubakar', Salim Rabi'u Kabara ${ }^{2}$, Umar Tasi'u Mustapha $^{3}$, Mujahid Guda Musa ${ }^{4}$ \\ ${ }^{1,2}$ Jodhpur National University, Rajasthan \\ ${ }^{3}$ Federal University, Dutse \\ ${ }^{4}$ Jodhpur National University, Rajasthan
}

\begin{abstract}
In this work, to show that real life cases scenario when tested satisfied queuing theory, we obtain data from kalinga restaurant, Jodhpur. We then calculated the arrival rate, service rate, utilization rate, waiting time and the probability of potential customers to balk based on the collected data, Little's theorem and M/M/I queuing model. The arrival rate during busiest period of the day is 1.42 customer per minute. The average number of customers in the restaurant is 70 and utilisation rate of 0.9859 . We conclude the work by given the concluding remarks.
\end{abstract}

Keywords: Queue, Little's theorem, exponential distribution

\section{Introduction}

There are many factors for a restaurant to attract customers, the most important factors are; taste, cleanliness, [5] food quality, service quality restaurant environment and setting. [2] These factors, when managed carefully, will be able to attract plenty of customers. Furthermore, there is another important factor that needs to be considered, that is queuing time. Queuing theory is the study of queue or waiting lines. Some of the analysis that can be derived using queuing theory includes the expected queue length, the probability of the system to be in certain states, such as empty or full [1].

\section{Queuing Theory}

In 1903 FrJohannsen was appointed copehengen telephone company director and he observed that the manual switch were not dimensioned in the right way. FrJohannsen published some work using the mathematical theory of probability from an economic point of view he stated that in one of his work "The overloading of the subscribers resulted in considerable extra expenses on account of the telephone operators having to make repeated attempt to establish a connection".

In order to develop more precise dimensioning FrJohannsen established scientific laboratory where he engaged the mathematician AgnerKrarupErlang. Erlang stated to work on the holding time in a telephone switch and then identified that the telephone conversations satisfied a Poisson distribution as well as the telephone holding time was exponentially distributed. Erlang work is the origin of the current network optimisation and queuing theory [6].

\section{Little's Theorem}

Little's theorem [3, 4] describes the relationship throughout arrival and service rate, cyclic time and work process. The theorem states that the expected numbers of customers $L$ for a system in steady state can be derive from little's theorem;

$$
L=\lambda T
$$

Where $\lambda$ is the average customers arrival rate and $T$ is the average service time for a customer.

Three fundamental relationships can be derive from little's theorem

- $L$ Increase if $\lambda$ or $T$ increase

- $\lambda$ Increase if $L$ increaseor $T$ decrease

- $T$ Increase if $L$ increase or $\lambda$ decrease

\section{M/M/1 Queuing Model}

$\mathrm{M} / \mathrm{M} / \mathrm{I}$ queuing model means that arrival and service time are exponentially distributed or Poisson process.

The following variables will be investigated;

- $\lambda$ : The mean customer's arrival rate

- $\mu$ : The mean service rate

$$
\rho=\frac{\lambda}{\mu}: \text { Utilization factor }
$$

- $P_{n}$ : The probability of having $n_{\text {customers }}$

$$
P_{n}=(1-\rho) \rho^{n}
$$

* Probability of zero customers

$$
P_{0}=1-\rho
$$

- $L$ : The average number of customers

$$
L=\frac{\rho}{1-\rho}=\frac{\lambda}{\mu-\lambda}
$$

- $L_{q}$ : The average number of customers in the queue

$$
L_{q}=L \times \rho=\frac{\rho^{2}}{1-\rho}=\frac{\rho \lambda}{\mu-\lambda} .
$$

- $W_{q}$ : The average waiting time in the queue 


\section{International Journal of Science and Research (IJSR) \\ ISSN (Online): 2319-7064}

Index Copernicus Value (2013): 6.14 | Impact Factor (2015): 6.391

$$
W_{q}=\frac{L_{q}}{\lambda}=\frac{1}{\mu-\lambda}
$$

- *W: The average time spent, including the waiting time

$$
W=\frac{L}{\lambda}=\frac{1}{\mu-\lambda}
$$

\section{Chart Title}

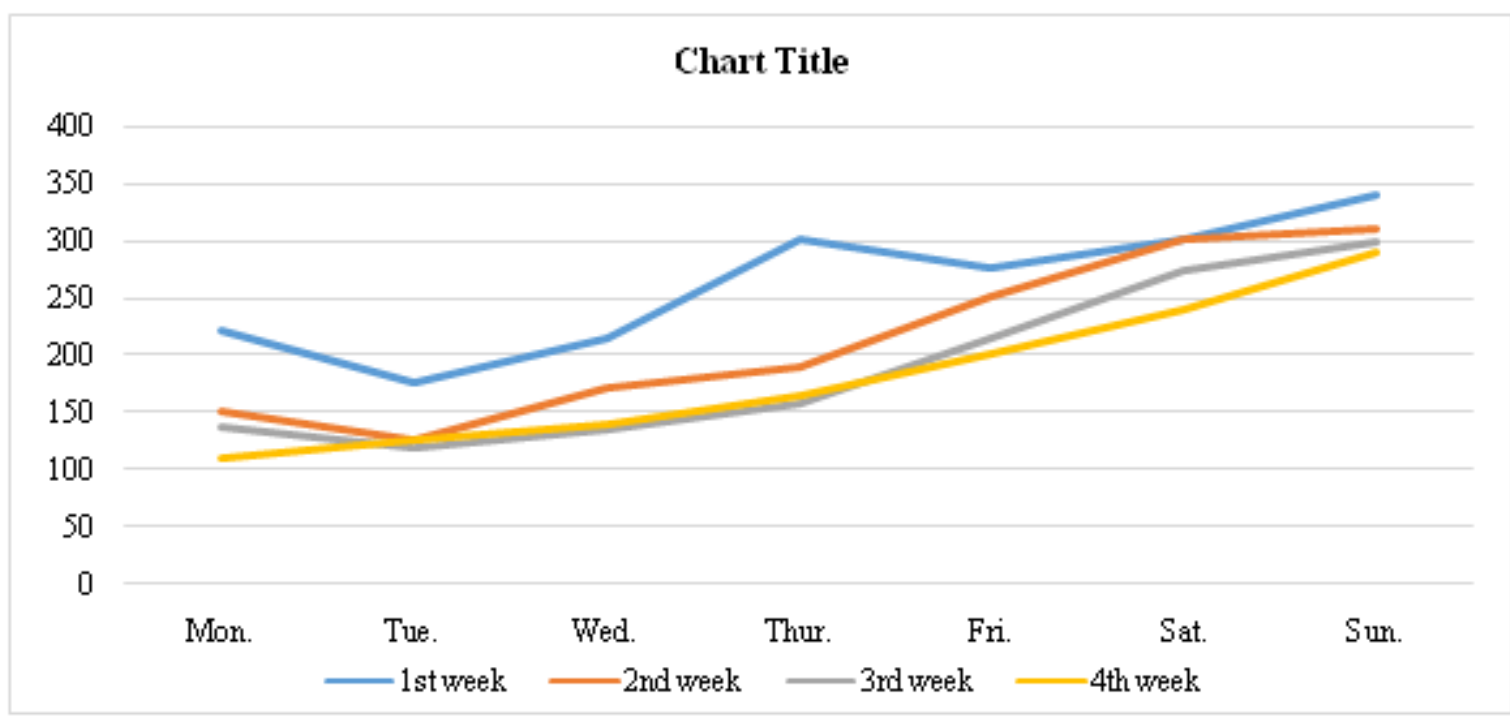

One month daily customers count

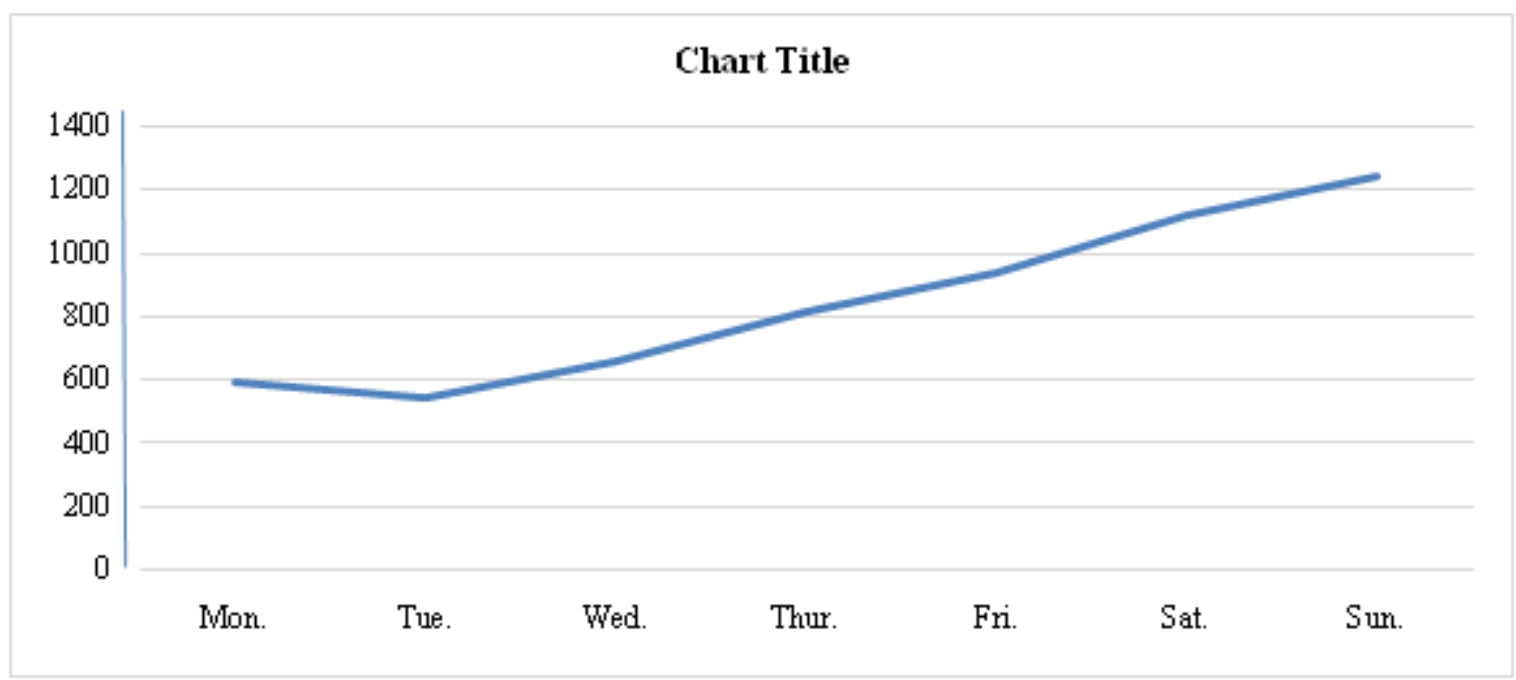

One month total Customers Count

\section{Calculations}

It was investigated that, after Friday, during the weekend there are on average 210 people coming to the restaurant in $2 \frac{1}{2}$ hours' time of dinner. From this we obtain the arrival rate as;

$\lambda=\frac{210}{150}$

$=1.4$ Customers $/$ minutes

\section{Observation}

Below is one month daily customer data which was collected from the restaurant

Table One month customer count

\begin{tabular}{|c|c|c|c|c|c|c|c|}
\hline & Mon. & Tues. & Wed. & Thur. & Fri. & Sat. & Sun. \\
\hline $1^{\text {st }}$ week & 200 & 175 & 215 & 300 & 275 & 300 & 340 \\
\hline $2^{\text {nd }}$ week & 150 & 125 & 170 & 190 & 250 & 300 & 310 \\
\hline $3^{\text {rd }}$ week & 137 & 119 & 134 & 157 & 215 & 275 & 300 \\
\hline $4^{\text {th }}$ week & 110 & 125 & 140 & 165 & 200 & 240 & 290 \\
\hline Total & 597 & 544 & 659 & 812 & 940 & 1115 & 1240 \\
\hline
\end{tabular}


Next, we will calculate the average number of people in the restaurant using (6)

$L=1.4$ cpm $\times 50 \mathrm{~min}$

$=70$ customers

Using (2) \& (5) we can also calculate the service and utilization rate as shown below;

$\mu=\frac{1.4(1+70)}{70}$

$=1.42$

Probability of customers going away $=\mathrm{P}$ (more than 10 people in the queue)

$=\mathrm{P}($ more than 110 people in the restaurant $)$

$\therefore P_{111-130}=\sum_{n=111}^{130} P_{n}$

$=0.01408 \sum_{n=111}^{130}(0.9859)^{n}$

$=0.051157132$

$=5.1157 \%$

\section{Concluding Remarks}

- The utilization rate at the restaurant is very large (0.9859). This is only utilization rate during lunch and dinner on Saturday and Sunday.

- In case the customer waiting time is less than $15 \mathrm{mins}$, the number of customers that will be served per minute will increase. When the service rate is higher the utilization rate will be lower.

- The queue model formulation is much simpler than to provide simulation model.

- This research can help to increase the quality of service by anticipating if there are many customers in the queue.

\section{References}

[1] Dharmawirya, M., \&Adi, E., "Case study for restaurant queuing model" 2011 international conference on management and artificial intelligence. IPEDR vol.6 (2011), pp. 52-55.

[2] Haghighi, M., Dorosti, A.,Rahnama, A., \&Hoseinpour, A. "Evaluation of factors affecting customer loyalty in restaurant industry". African Journal of Business Management, vol. 6(14), April, 2012, pp. 5041.

[3] Little, J.D.C., "A proof for the queuing formula $L=\lambda W$ " Operations Research, vol. 9(3), 1961, pp. 383-387, doi: 10.2307/167570.

[4] Little, J.D.C., “Little's law as viewed on its $50^{\text {th }}$ anniversary" Operations Research vol. 59, No. 3 MayJune 2011, pp. 536-549.

[5] Mishra, G.D., Chauhan, V.S., \& Chandra, N. "A study of service in restaurant by using queuing model". The Bulletin of society for mathematical service and standards vol. 5 (2013), pp. 14-18.

[6] Stardahl, K. "The History behind the Probability Theory and Queuing Theory" elekronikk 2.2007. 